\title{
Modified Distal Urethral Polypropylene Sling (Canal Transobturator Tape) Procedure: Efficacy for Persistent Stress Urinary Incontinence After a Conventional Midurethral Sling Procedure
}

\author{
Chang Hee Kim, Tae Beom Kim, Jin Kyu Oh, Sang Jin Yoon, Khae Hawn Kim, Kwang Taek Kim \\ Department of Urology, Gachon University Gil Medical Center, Gachon University of Medicine and Science, Incheon, Korea
}

\begin{abstract}
Purpose: Despite reports of persistent stress urinary incontinence (SUI) in patients after the midurethral sling (MUS) procedure, there is no widely accepted definition or cause of the condition. In many cases, the mesh implanted in the previous MUS procedure has been found to have migrated proximally. The aim of this study was to evaluate the efficacy of the modified distal urethral polypropylene sling, or canal transobturator tape (TOT), procedure for persistent SUI after a conventional MUS procedure on the assumption that persistent SUI after MUS is due to the location of the sling.

Methods: From January 2008 to April 2012, 31 female patients who underwent the canal TOT procedure presented with incontinence or lower urinary tract symptoms (LUTS) were included in this study. We identified patients who had been operated on by use of the conventional MUS procedure at other medical facilities, whose Valsalva leak pressure point was less than $120 \mathrm{~cm}$ $\mathrm{H}_{2} \mathrm{O}$ by urodynamic study, and who were also diagnosed with persistent $\mathrm{SUI}$. If vaginal or urethral mesh exposure was concomitant with persistent SUI, the mesh was removed completely or in part. Surgical procedures for canal TOT were identical to the original TOT procedures, except in the number and location of the vaginal incisions. Incontinence Impact Questionnaire-Short Form (IIQ-7) and Urogenital Distress Inventory-Short Form (UDI-6) scores were assessed preoperatively and at 3 months postoperatively.

Results: There were no intraoperative or postoperative complications. Twenty-eight patients (90.3\%) showed improvement in incontinence or other LUTS. Postoperative scores of the IIQ-7 (0.65 \pm 0.48$)$ and UDI-6 (3.48 \pm 2.28$)$ were significantly improved compared with preoperative scores $(1.26 \pm 0.58$ and $7.52 \pm 4.30$, respectively; $\mathrm{P}<0.05)$.

Conclusions: Improper sling location is one of the major causes of persistent SUI after the conventional MUS procedure. Our results demonstrate that canal TOT may be an alternative method in the treatment of persistent SUI after the conventional MUS procedure.
\end{abstract}

Keywords: Stress urinary incontinence; Suburethral slings; Recurrence

\section{INTRODUCTION}

Since Ulmsten described the tension-free vaginal tape (TVT)

Corresponding author: Kwang Taek Kim

Department of Urology, Gachon University Gil Medical Center,

Gachon University of Medicine and Science, 21 Namdong-daero 774beon-gil,

Namdong-gu, Incheon 405-760, Korea

Tel: +82-32-460-3334 / Fax: +82-32-460-8340 / E-mail: shinekkt@gmail.com

Co-corresponding author: Khae Hawn Kim

Department of Urology, Gachon University Gil Medical Center,

Gachon University of Medicine and Science, 21 Namdong-daero 774beon-gil,

Namdong-gu, Incheon 405-760, Korea

Tel: +82-32-460-3334 / Fax: +82-32-460-8340,

E-mail: kimcho99@gilhospital.com

Submitted: March 7, 2013 / Accepted after revision: March 26, 2013 procedure in 1995, midurethral synthetic sling (MUS) procedures, including the transobturator tape (TOT) procedure, have grown in acceptance and popularity for stress urinary inconti-

This is an Open Access article distributed under the terms of the Creative Commons Attribution Non-Commercial License (http://creativecommons.org/licenses/by-nc/3.0/) which permits unrestricted non-commercial use, distribution, and reproduction in any medium, provided the original work is properly cited. 
nence (SUI) surgery. These procedures have become the new "gold standard" for the surgical treatment of female SUI, not only because of their simplicity for both the surgeon and the patient, but also because of positive surgical outcomes and low morbidity. However, $5 \%$ to $23 \%$ of patients will have persistent or recurrent SUI after a MUS procedure [1,2]. Generally, when persistent or recurrent SUI occurs after a MUS procedure, it is considered to be a failure of treatment or misdiagnosis. Urodynamic study (UDS) is useful to distinguish between failure of treatment and misdiagnosis. Although no consensus exists regarding treatment failure, the position of the tape relative to the proximal urethra has been investigated as a cause of recurrent SUI after MUS. Therefore, many procedures have been performed to treat patients with persistent or recurrent SUI after MUS, such as redo MUS, implantation of an artificial urethral sphincter, injection of a periurethral bulking agent, use of a pubovaginal sling, or shortening of the tape.

We posited that persistent or recurrent SUI after a MUS procedure is due to the inadequate location of a sling. Thus, we performed a modified distal urethral polypropylene sling procedure, also known as canal TOT, in which we placed the sling more distally in cases of persistent or recurrent SUI after conventional MUS. The aim of this study was to evaluate the efficacy of this modified procedure after we confirmed by UDS that no misdiagnosis had occurred.

\section{MATERIALS AND METHODS}

Between January 2008 and April 2012, 31 patients who had been diagnosed with persistent SUI underwent a canal TOT procedure. If a patient had consistent or recurrent lower urinary tract symptoms (LUTS) even after an MUS procedure at another facility and a Valsalva leak point pressure (VLPP) less than $120 \mathrm{cmH}_{2} \mathrm{O}$ by UDS, the patient was diagnosed with persistent SUI. The patients' medical histories and results of the physical examination, urinalysis, urine culture, and UDS, including VLPP, were evaluated as part of the preoperative preparation.

The Incontinence Impact Questionnaire-Short Form (IIQ-7) and Urogenital Distress Inventory-Short Form (UDI-6) were used to compare the patients' subjective satisfaction before and after the canal TOT procedure [1,2]. All statistical analyses were performed by using SPSS ver. 15.0 (SPSS Inc., Chicago, IL, USA). Paired t-tests were used to compare preoperative and postoperative IIQ-7 and UDI-6 scores. P-values less than $0.05(\mathrm{P}<0.05)$ were considered statistically significant.
A trained one surgeon performed all operations. Patients were placed into the dorso-lithotomy position under spinal or general anesthesia. The operation was begun with removal of the tape that had previously been inserted during MUS. With the use of a vaginal weighted retractor and hook retractor, a clear view against the vaginal wall was secured and the tape was found with careful palpation (Fig. 1A). To confirm placement of the previously inserted tape, a Foley catheter was placed into the neck of the bladder and inflated with $10 \mathrm{~mL}$ of sterile water. A midline incision was then made into the anterior vaginal wall mucosa covering the tape (Fig. 1B). The middle part of the tape was cut and trimmed laterally through the midline incision (Fig. 1C). The incision was closed with absorbable suture material after the tape was removed in whole or in part (Fig. 1D). Canal TOT was then performed by using the technique previously described in the literature [3,4]. Two, $1.5-\mathrm{cm}$ oblique lateral incisions were made in the anterior vaginal wall $1.5 \mathrm{~cm}$ below the external urethral meatus (Fig. 1E). Dissection was carried toward the ipsilateral shoulder through the lateral incisions to visualize the periurethral fascia. After a suburethral canal was created $1 \mathrm{~cm}$ below the urethra of the anterior vaginal wall by using a right angle clamp (Fig. 1F), it was transferred underneath the suburethral canal (Fig. 1G). Because the mesh is placed in a the suburethral canal, displacement of the mesh does not often occur after surgery. Two, $0.5-\mathrm{cm}$ incisions were then made approximately $0.5 \mathrm{~cm}$ medial to the genitocrural fold at the level of the clitoris on each side. An index finger was passed through the opening of the incision in the anterior vaginal wall and placed on the obturator membrane. A helical trocar was inserted from the incision in the genitocrural fold and passed through the obturator membrane. The tip of the trocar was drawn out toward the anterior vaginal wall incision with careful palpation (Fig. 1H). The end of the tape was introduced into the eye of the trocar and was pulled out toward the incision in the genitocrural fold. An Allis forcep was placed through each of the two incisions of the anterior vaginal wall and the tape was clamped. Excess tape on both sides of the genitocrural fold area was trimmed while the tape was maintained free of tension. Incisions of the anterior vaginal wall were sutured with absorbable suture material. Finally, vaginal packing with povidone-iodine-saturated gauzes was applied. When cystocele repair was needed, an additional incision was made proximal to the sutured incision on the anterior vaginal wall to the middle of the anterior vaginal wall. On postoperative day 1 (hospital day 3), the Foley catheter inserted during the procedure was re- 


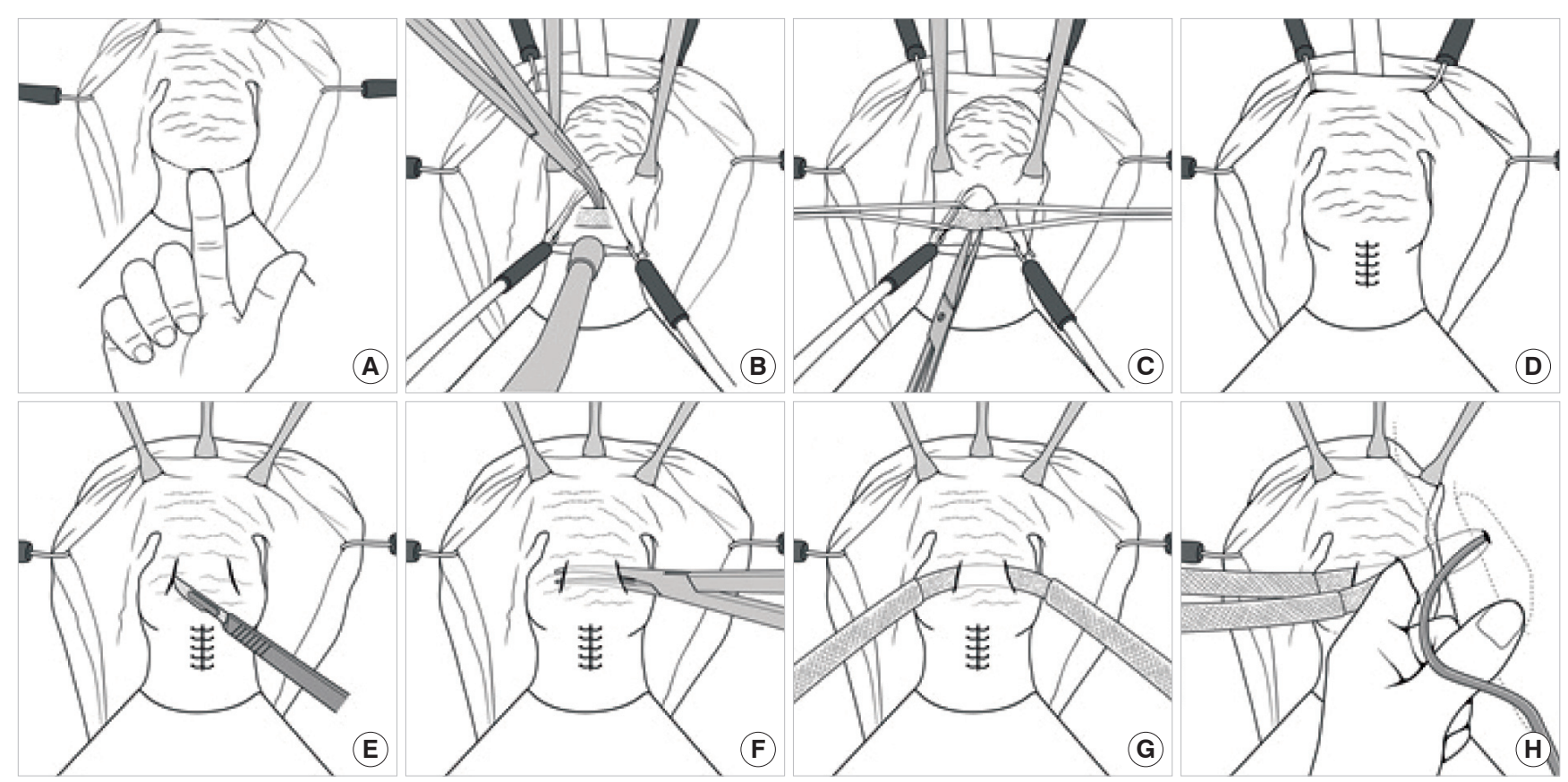

Fig. 1. (A) With the use of a vaginal weighted retractor and hook retractor, a view against the vaginal wall was secured and the tape was found with careful palpation. (B) A midline incision was then made into the anterior vaginal wall mucosa covering the tape. (C) The middle part of the tape was cut and trimmed laterally through the midline incision. (D) The incision was closed with absorbable suture material after the tape was removed in whole or in part. (E) $1.5-\mathrm{cm}$ oblique lateral incisions are made in the anterior vaginal wall. (F) A suburethral canal is created bluntly between the lateral incisions by using a fine, right angle clamp. The canal is located in the layer between the periurethral fascia and the urethra. (G) Monofilament polypropylene tape is transferred beneath the suburethral canal. $(\mathrm{H})$ Bilateral paraurethral dissection of the vagina is performed in the direction of the ischiopubic ramus through the dissected lateral incision. The tunneler is passed through the obturator membrane. Its tip is palpated with the index finger on the medial side of the obturator membrane by paraurethral dissection.

moved and most patients were discharged.

\section{RESULTS}

The average age of the patients was $51.90 \pm 7.54$ years (range, 31 to 67 years). The average number of vaginal births was $2.12 \pm 0.78$ (range, 1 to 4). The average time interval between MUS and canal TOT was $16.96 \pm 14.29$ months (range, 6 to 48 months). Average VLPP was $58.90 \pm 14.10 \mathrm{cmH}_{2} \mathrm{O}$, and the detrusor pressure at maximal flow rate was $29.50 \pm 12.29 \mathrm{cmH}_{2} \mathrm{O}$. One patient underwent the TVT procedure. A total of 30 patients had the TOT operation (TVT-O included) (Table 1). The average operation time was $40.40 \pm 7.20$ minutes (range, 30 to $60 \mathrm{~min}$ utes), excluding the time for other procedures. Average hospitalization was $3.36 \pm 0.57$ days (range, 3 to 5 days) (Table 2). In some cases, other procedures were performed at the time of canal TOT: 13 cases of vaginal tape erosion repair (42\%), 2 cases of urethral tape erosion repair (6\%), 1 case of vaginal mass exci- sion (3\%), 1 case of bladder stone removal (3\%), 3 cases of cystocele repair (10\%), and 1 case of rectocele repair (3\%) (Table 2). There were no postoperative complications such as bladder, bowel, or vascular injury or the need for transfusion owing to excessive bleeding. There were no urination-related complications except for three cases of transient, de novo urgency (Table 2). Continence was maintained in 28 cases after canal TOT (90\%). Each questionnaire revealed statistically significant improvement from before to 3 months after canal TOT. The IIQ-7 and UDI-6 scores were decreased significantly from $1.26 \pm 0.58$ to $0.65 \pm 0.48(\mathrm{P}<0.05)$ and from $7.52 \pm 4.30$ to $3.48 \pm 2.2(\mathrm{P}<$ $0.05)$, respectively (Table 3 ).

\section{DISCUSSION}

According to the "hammock hypothesis," continence is preserved when urethral closing pressure increases following an increase in abdominal pressure owing to compression of the 
Table 1. Preoperative patient characteristics

\begin{tabular}{lc}
\hline Characteristic & Value \\
\hline Age $(\mathrm{yr})$ & $51.9(31-67)$ \\
No. of vaginal deliveries & $2.12(1-4)$ \\
Duration after previous operation $(\mathrm{mo})$ & $16.96(6-48)$ \\
Urodynamic study characteristics $\left(\mathrm{cmH}_{2} \mathrm{O}\right)$ & \\
Valsalva leak pressure point & $58.90(44.80-73.00)$ \\
PdetQmax & $29.50(17.20-41.80)$ \\
Previous incontinence surgery & \\
Transobturator tape & $30(97)$ \\
Tension-free vaginal tape & $1(3)$ \\
No. of incontinence & $18(58)$ \\
Pure stress & $13(32)$ \\
Mixed type & \\
\hline
\end{tabular}

Values are presented as mean (range) or number (\%).

PdetQmax, the detrusor pressure at maximal flow rate.

Table 3. Preoperative and postoperative IIQ-7 and UDI-6 results

\begin{tabular}{lcclcc}
\hline & \multicolumn{2}{c}{ Preoperation } & & \multicolumn{2}{c}{ Postoperation } \\
\cline { 2 - 3 } \cline { 5 - 6 } & Mean $\left.( \pm \text { SD })^{\mathrm{a}}\right)$ & Range & & Mean $\left.( \pm \mathrm{SD})^{\mathrm{a}}\right)$ & Range \\
\hline IIQ-7 & $1.26( \pm 0.58)$ & $0.43-2.43$ & & $0.65( \pm 0.48)$ & $0-2.14$ \\
UDI-6 & $7.52( \pm 4.30)$ & $1-16$ & & $3.48( \pm 2.28)$ & $0-8$ \\
\hline
\end{tabular}

IIQ-7, Incontinence Impact Questionnaire-Short Form; UDI-6, Urogenital Distress Inventory-Short Form; SD, standard deviation.

${ }^{\text {a) }} \mathrm{P}<0.05$, paired $t$-test.

urethra by its hammock-like supportive layer [5]. Ulmsten first introduced the TVT procedure in 1996 on the basis of the integral theory [6]. Since then, the MUS has come into widespread use and has shown excellent success rates in long-term studies. However, complications after surgery soon came to the fore; these can include infection of the wound or urinary tract, bladder perforation, injury to the bowel, or vascular-, nerve-, and urinary-related complications. Thus, the transobturator route method, which does not pass through the retro-pubic route, was devised. The TOT procedure is commonly used to treat female patients with SUI owing to its safety and effectiveness. However, $5 \%$ to $23 \%$ of patients will have persistent or recurrent SUI after MUS (TOT) [1]. Even though redo TOT is performed in many cases owing to persistence or recurrence of incontinence after a TOT operation, the cause of relapse has yet to be defined.

When urine leakage is persistent or recurrent within a cer-
Table 2. Operative data

\begin{tabular}{lc}
\hline Variable & Value \\
\hline Postoperative results (sling only) & \\
Operation time (min) & $40.40(30-60)$ \\
Hemoglobin change (g/dL) & $0.7(0.5-0.9)$ \\
Hospital stay (day) & $3.36(3-5)$ \\
Follow-up (total) (mo) & $3.8(1.9-5.7)$ \\
Concomitant procedure & \\
Sling alone & $10(32)$ \\
Cystocele repair & $3(10)$ \\
Rectocele repair & $1(3)$ \\
Anterior vaginal wall mass excision & $1(3)$ \\
Repair of urethral tape erosion & $2(6)$ \\
Repair of vaginal tape erosion & $13(42)$ \\
Bladder stone removal & $1(3)$ \\
Intraoperative and short-term complications & \\
Bladder perforation & $0(0)$ \\
De novo urgency (transient) & $3(10)$ \\
Weak stream & $0(0)$ \\
Hematoma in Retzius' space & $0(0)$ \\
Difficult emptying & $0(0)$ \\
Long-term complications & $0(0)$ \\
Inguinary obstruction & $0(0)$ \\
Mesh erosion & $0(0)$ \\
Dyspareunia & \\
\hline
\end{tabular}

Values are presented as mean (range) or number (\%).

tain period of time after an incontinence procedure, the operation is regarded as a failure. In this case, the urine leakage is commonly termed persistent SUI. However, the exact etiology has not been identified. The cause of persistent SUI is also unclear; detrusor overactivity or intrinsic sphincter deficiency (ISD) could be one cause. A loosely placed tape or a tape that is not located in the midurethra precisely during surgery, infection, or erosion could also be postsurgical causes of persistent SUI. In this study, persistent SUI was defined as the presence of LUTS, including urine leakage, after MUS and a VLPP less than 120 $\mathrm{cmH}_{2} \mathrm{O}$. Medical history, physical examination, urinalysis, urine culture, cystoscopy, UDS, and a preoperative exam were done for all 31 patients with persistent SUI in this study. Thus, we were able to eliminate infection and detrusor instability as causes of the persistent SUI after MUS; mean VLPP in our 
study was $85.90 \pm 13.10 \mathrm{cmH}_{2} \mathrm{O}$.

The sling location was reported to be important for treatment success in four case reports of persistent incontinence after a pubovaginal sling operation. According to these reports, if a sling is placed at the base of the bladder, it not only fails to restore continence, but in fact may worsen incontinence [7]. In another study, when a TVT was positioned between the 50th and the 80th percentile of urethral length, as measured from the bladder neck, the cure rate exceeded $90 \%$, whereas a position outside this zone was associated with treatment failure in $36 \%$ of patients [8]. Correspondingly, positioning of the TOT under the proximal half of the urethra increases failure risk 6 -fold [9]. Other researchers reviewed the outcomes of 98 women who underwent the TVT procedure and postoperative transvaginal ultrasonography to identify the sling location. Eleven women had persistent SUI postoperatively, and failure was associated with bladder neck funneling and a more proximal tape location in relation to the midurethra [9]. There are reports that over $70 \%$ of patients with persistent SUI after TVT had slings under the proximal half of the urethra. In a followup study, the authors described their technique of using twopoint suture fixation of the sling at the midurethra to prevent migration of the sling. Rates of cure and improvement in those undergoing tape fixation were significantly greater than in those with no fixation (95\% vs. $89 \%$ ) [10]. Thus, exact placement of a sling is the most important factor in MUS.

The location of the tape used in a previous surgery is confirmed by palpation of the graft through physical examination, manipulation of an inserted Foley catheter, transillumination through cystourethroscopy, or transvaginal ultrasonography. In our study, an inserted Foley catheter was used to confirm the location of the tape used in the previous surgery, and the location was reconfirmed by exploration of the anterior vaginal wall. In all 31 patients, the tape was located in the area of the bladder neck or proximal urethra.

A previous study showed one cause of persistent SUI after a pubovaginal sling procedure is the location of the sling. According to that study, 4 patients presented with a fixed open bladder neck and inadequate urethral coaptation on fluoroscopy, with the pubovaginal sling identified proximal to the bladder neck in each case intraoperatively. In other words, proximal placement of the sling resulted in fixation of the bladder neck in an open configuration [7].

Several treatment options are available for persistent SUI after MUS procedures, such as retropubic suspension, urethral sphincter, or repeat MUS [11]. Even though a study showed that a repeat synthetic MUS procedure has a significantly lower cure rate than a primary MUS procedure, repeat MUS is still used as an attractive option for initial MUS failure, because the MUS procedure is simple and has a high primary success rate [12].

A new combination surgical procedure, canal TOT, has been introduced and has shown a high success rate and a low number of complications [3,4]. By integration of the distal urethral polypropylene sling with the original TOT, the occurrence of voiding dysfunction such as de novo urgency can be reduced after SUI surgery [13].

The concept of the distal urethral complex was first introduced in 2003 [14]. The distal urethral complex is composed of the levator and other muscles that are connected to the pubourethral ligaments, compressor urethrae, urethrovaginal sphincter, and the distal urethra posterior wall. Researchers think that this complex regulates passive and active tension in the urethra $[14,15]$. The distal urethral sling supports the posterior part of the urethra, because the distal urethra is more proximal to the pubis. Thus, it increases partial resistance in the distal urethra. It also increases the supportive efficacy of the levator muscle and midurethra; the distal urethra and bladder neck are maintained at the same time in the urethra when abdominal pressure is increased. The distal urethral polypropylene sling has applied the concept of the distal urethral complex to the area of the posterior pubis. Canal TOT has changed the location of this concept from the posterior pubis to the obturator foramen and has been shown to have fewer side effects compared with the other procedure. For example, de novo urgency, a form of dysuria after surgery that contributes to negative satisfaction after surgery, has been reported with only a $3 \%$ frequency after canal TOT [3].

Gilchrist et al. [11] have described that the vertical trajectory of the mesh passing into the retropubic space might predispose the sling to a more proximal location, compared with the more horizontal trajectory of TOT arms via the obturator foramen [11]. According to Lee et al. [3], in canal TOT, displacement of the mesh does not often occur after surgery, because the mesh is placed in a tunnel made between two incisions in the anterior wall of the vagina. Thus, dysuria accompanied by SUI occurs far less than after other operations because the location of the mesh has been moved from the midurethra to the bladder neck [4].

In this study, we performed redo canal TOT in 31 patients with persistent SUI after MUS surgery. Among this group, 30 
patients had TOT surgery and 1 patient had TVT surgery. Even though 3 patients out of 31 who underwent redo canal TOT experienced transient, de novo urgency, there was no long-term dysuria or any other postoperative side effects, such as bladder, bowel, or vascular injury or the need for transfusion due to excessive bleeding. There was also no dysuria accompanied by SUI due to displacement of the mesh to the bladder neck. Also, the symptoms of 3 patients with transient de novo urgency were improved in 3 months. Most patients achieved continence and showed statistically significant improvement in UDI-6 and IIQ7 scores.

A limitation of the present study is that the follow-up period was only 6 to 48 months after canal TOT for recurrent SUI after MUS. Because comparative analysis between preexisting methods and canal TOT and long-term follow-up were not fully established, further study is needed.

Even though several causes of recurrent SUI after MUS are possible, the one of the most important causes is the location of the sling. Thus, the location of the sling should be examined in patients with LUTS after MUS. In this study, to treat recurrent SUI after MUS, we performed canal TOT and obtained valuable results. The patients almost never mentioned complications after the surgery. The symptoms of SUI and discomfort in everyday life were significantly decreased from a subjective satisfaction point of view. As our results show, canal TOT can be an alternative treatment in recurrent SUI after MUS because of the location of the sling, i.e., proximal to bladder neck.

\section{CONFLICT OF INTEREST}

No potential conflict of interest relevant to this article was reported.

\section{REFERENCES}

1. Ogah J, Cody DJ, Rogerson L. Minimally invasive synthetic suburethral sling operations for stress urinary incontinence in women: a short version Cochrane review. Neurourol Urodyn 2011;30:284-91.

2. Daneshgari F, Kong W, Swartz M. Complications of mid urethral slings: important outcomes for future clinical trials. J Urol 2008;180: 1890-7.

3. Lee SH, Kim TB, Kim KH, Jung H, Lee MS, Yoon SJ. Preliminary report on a modified surgical technique using canal transobturator tape for the treatment of female stress urinary incontinence. Korean J Urol 2008;49:1119-24.

4. Lee JH, Yoon HJ, Lee SJ, Kim KH, Choi JS, Lee KW. Modified transobturator tape (canal transobturator tape) surgery for female stress urinary incontinence. J Urol 2009;181:2616-21.

5. DeLancey JO. Structural support of the urethra as it relates to stress urinary incontinence: the hammock hypothesis. Am J Obstet Gynecol 1994;170:1713-20.

6. Ulmsten U, Henriksson L, Johnson P, Varhos G. An ambulatory surgical procedure under local anesthesia for treatment of female urinary incontinence. Int Urogynecol J Pelvic Floor Dysfunct 1996; 7:81-5.

7. Poon C, Zimmern P. When the sling is too proximal: a specific mechanism of persistent stress incontinence after pubovaginal sling placement. Urology 2004;64:287-91.

8. Kociszewski J, Rautenberg O, Perucchini D, Eberhard J, Geissbuhler V, Hilgers R, et al. Tape functionality: sonographic tape characteristics and outcome after TVT incontinence surgery. Neurourol Urodyn 2008;27:485-90.

9. Yang JM, Yang SH, Yang SY, Yang E, Huang WC, Tzeng CR. Clinical and pathophysiological correlates of the symptom severity of stress urinary incontinence. Int Urogynecol J 2010;21:637-43.

10. Rechberger T, Futyma K, Jankiewicz K, Adamiak A, Bogusiewicz M, Bartuzi A, et al. Tape fixation: an important surgical step to improve success rate of anti-incontinence surgery. J Urol 2011;186: 180-4.

11. Gilchrist AS, Rovner ES. Sling location in women with recurrent stress urinary incontinence following midurethral sling. Urology 2012;79:76-9.

12. Scarpero HM, Dmochowski RR. Sling failures: what's next? Curr Urol Rep 2004;5:389-96.

13. Rutman M, Itano N, Deng D, Raz S, Rodriguez LV. Long-term durability of the distal urethral polypropylene sling procedure for stress urinary incontinence: minimum 5-year followup of surgical outcome and satisfaction determined by patient reported questionnaires. J Urol 2006;175:610-3.

14. Rodriguez LV, Raz S. Prospective analysis of patients treated with a distal urethral polypropylene sling for symptoms of stress urinary incontinence: surgical outcome and satisfaction determined by patient driven questionnaires. J Urol 2003;170:857-63.

15. Rodriguez LV, Raz S. Polypropylene sling for the treatment of stress urinary incontinence. Urology 2001;58:783-5. 